TENDENCIAS

Revista de la Facultad de Ciencias

Económicas y Administrativas.

Universidad de Nariño

ISSN-E 2539-0554

Vol. XXII No. 2 - 2do Semestre 2021

Julio-Diciembre - Páginas 76-106

\title{
HACIA UN TURISMO INCLUSIVO EN LOS HOTELES COMERCIALIZADOS POR ECOTUR EN LA HABANA Y VARADERO
}

TOWARDS INCLUSIVE TOURISM IN THE HOTELS MARKETED BY ECOTUR IN HAVANA AND VARADERO

\section{RUMO AO TURISMO INCLUSIVO NOS HOTÉIS MARCADOS PELA ECOTUR EM HAVANA E VARADERO}

Félix Hernández Domínguez; Yoan Hernández Flores; Maité Rodríguez González

Licenciado en Turismo, Universidad de La Habana. Adiestrado en Gestión de Operaciones, Departamento Plaza II, Palmares Centro, OSDE Cubasol. ORCiD: 0000-0002-5669-7383. E-mail: felixdguez96@gmail.com, Cuba.

Licenciado en Turismo, Universidad de La Habana. Docente e Investigador, Facultad de Turismo, Universidad de La Habana. ORCiD: 0000-0003-1935-2594. E-mail: yoan.hernandez@ftur.uh.cu, Cuba.

Máster en Gestión Turística, Universidad Central Marta Abreu de las Villas. Especialista III en Investigación Innovación y Desarrollo, Instituto de Investigaciones de Ingeniería Agrícola (INIFAT). ORCiD: 0000-0002-5685-457X. E-mail: maiterdguez83@gmail.com, Cuba.

Recibido: 7 de febrero de 2021

Aprobado: 26 de abril de 2021

DOI: https://doi.org/10.22267/rtend.212202.169

\section{Resumen}

Emprender acciones a partir de la evaluación de problemas funcionales y de infraestructura en cada establecimiento hotelero en los destinos, constituye un punto de partida para elevar su nivel 
de accesibilidad, lo cual puede contribuir finalmente al diseño de productos turísticos más adecuados y en correspondencia con las necesidades de los diferentes segmentos de demanda. A partir de las necesidades de la agencia de viajes Ecotur, se realiza la presente investigación, la cual tiene como objetivo diagnosticar el nivel de accesibilidad de los hoteles comercializados por esta agencia en La Habana y Varadero. Para ello se utilizaron técnicas y herramientas como el análisis bibliográfico - documental, la observación directa, la encuesta y el análisis porcentual. La evaluación de los hoteles se realizó a partir de un procedimiento validado por expertos con el uso del método Delphi. Como principales resultados se obtuvieron el predominio del nivel alto de accesibilidad hotelera, sin diferencias entre ambas ciudades, en el acceso principal al recinto, comunicación vertical, áreas comunes y habitaciones adaptadas; influyeron negativamente la accesibilidad exterior y la formación del personal. Se proponen un total de 12 acciones para elevar los índices de accesibilidad en los hoteles objeto de estudio.

Palabras clave: accesibilidad; acciones; evaluación; hoteles; infraestructura; problemas funcionales.

JEL: A12; A14; L80; M10; M21; M54

\begin{abstract}
Taking actions based on the evaluation of functional and infrastructure problems in each hotel establishment in the destinations, constitutes a starting point to increase their level of accessibility, which can finally contribute to the design of more appropriate tourism products and in correspondence with the needs of the different demand segments. Based on the needs of the Ecotur travel agency, the present investigation is carried out, which aims to diagnose the level of accessibility of the hotels marketed by this agency in Havana and Varadero. For this, techniques and tools such as bibliographic-documentary analysis, direct observation, survey and percentage analysis were used. The evaluation of the hotels was carried out from a procedure validated by experts with the use of the Delphi method. The main results were the predominance of the high level of hotel accessibility, without differences between both cities, in the main access to the enclosure, vertical communication, common areas and adapted rooms; exterior accessibility and staff training had a negative influence. A total of 12 actions are proposed to increase accessibility rates in the hotels under study.
\end{abstract}


Keywords: actions; accessibility; evaluation; hotels; infrastructure; functional problems. JEL: A12; A14; L80; M10; M21; M54

\section{Resumo}

A realização de ações com base na avaliação dos problemas funcionais e de infraestrutura em cada estabelecimento hoteleiro dos destinos, constitui um ponto de partida para aumentar o seu nível de acessibilidade, podendo finalmente contribuir para a conceção de produtos turísticos mais adequados e em correspondência com as necessidades dos diferentes segmentos de demanda. Com base nas necessidades da agência de viagens Ecotur, realiza-se a presente investigação, que visa diagnosticar o nível de acessibilidade dos hotéis comercializados por esta agência em Havana e Varadero. Para tanto, foram utilizadas técnicas e ferramentas como análise bibliográficadocumental, observação direta, survey e análise percentual. A avaliação dos hotéis foi realizada a partir de procedimento validado por especialistas com a utilização do método Delphi. Os principais resultados foram a predominância do alto nível de acessibilidade hoteleira, sem diferenças entre as duas cidades, no acesso principal ao recinto, comunicação vertical, áreas comuns e quartos adaptados; a acessibilidade exterior e a formação do pessoal influenciaram negativamente. Ao todo, são propostas 12 ações para aumentar as taxas de acessibilidade nos hotéis em estudo.

Palavras-chave: ações; acessibilidade; avaliação; hotéis; infraestructura; problemas funcionais. JEL: A12; A14; L80; M10; M21; M54

\section{Introducción}

En las sociedades de todo el mundo, hablar de discapacidad es hablar de uno de los grandes problemas sociales. Aunque se manejan los términos dentro de procesos de inclusión, protección

y prevención, no es menos cierto que en países en vías de desarrollo todavía no se da la debida importancia al problema (Gordillo y Pérez, 2015).

La Convención sobre los Derechos de las Personas con Discapacidad (CDPD), adoptada en 2006, define a las personas con discapacidad como "aquellas que tienen deficiencias físicas, mentales, intelectuales o sensoriales a largo plazo y que, al interactuar con diversas barreras, pueden ver 
impedida su participación plena y efectiva en la sociedad, en igualdad de condiciones con los demás" (Organización Mundial del Turismo [OMT], 2015).

Según el informe del Banco Mundial (2019) “unos 1000 millones de habitantes, o sea el $15 \%$ de la población del mundo, experimentan algún tipo de discapacidad, y la prevalencia de la discapacidad es mayor en los países en desarrollo" (p.1). Este análisis realizado señala que, entre 110 millones y 190 millones de personas, o sea la quinta parte del total de habitantes en el mundo, experimentan discapacidad considerable, resultado de la interacción entre las características del organismo humano y las barreras actitudinales y del entorno que evitan su participación plena y efectiva en la sociedad, en igualdad de condiciones.

Esta situación se acentúa cuando el mundo se ha visto envuelto en un continuo proceso de interconexiones complejas y dinámicas capaz de generar nuevos escenarios susceptibles, y a su vez, de producir nuevas relaciones sociales. El logro de la integración a la sociedad de todas las personas con necesidades asociadas o no las discapacidades en un entorno más inclusivo y accesible, depende del nivel de concientización y el cambio de todos los miembros de la sociedad en su conjunto (Zurita, 2018).

El alto nivel de progreso alcanzado en nuestros días ha dado lugar a la conexión de dos fenómenos que hasta hace tan solo un par de décadas, era prácticamente inimaginable: Turismo y Discapacidad (Clemente, 2015 y Cruces, 2016).

A ese entramado general compuesto por las prácticas, las relaciones, los procesos y los discursos oficiales en torno a la actividad turística de las personas con discapacidad, se le ha llamado Turismo Accesible. Aunque existen otras denominaciones usadas en un tono más cercano al marketing social como Turismo Inclusivo o Turismo para todos, que se refieren al mismo fenómeno (Cruces, 2016).

Vinculado a ello, inicialmente el término de turismo accesible se encontraba asociado a la participación de personas con discapacidad en la oferta turística, y la eliminación de las barreras 
existentes en los diferentes ámbitos, espacios y servicios del sector turístico (Marcos y González, 2003; Buhalis y Darcy, 2011; OMT, 2014; Gordillo y Pérez, 2015).

Sin embargo, Bernaza (2019) plantea que el término ha sido ampliado teniendo en cuenta no sólo a las personas con discapacidad, sino a todos los individuos, independientemente de sus condiciones, ya que no sólo las personas con alguna discapacidad se benefician de la accesibilidad en el turismo, también lo hacen, por ejemplo, familias con niños pequeños, personas con discapacidad transitoria, embarazadas, el grupo poblacional que pertenece a la tercera edad, entre otros (Porto y Rucci, 2019). Es por ello, que los gestores de los destinos turísticos, deben garantizar la infraestructura necesaria para todas las personas.

En este sentido afirma Rodríguez (2017) que "la accesibilidad de los entornos y servicios contribuye a mejorar la calidad del producto turístico e incrementa la competitividad general de los destinos (...) debe, por consiguiente, ser parte intrínseca de cualquier política y estrategia de turismo responsable y sostenible" (p.114).

Poner en marcha iniciativas de Turismo Accesible implica un conjunto de actuaciones, previas y durante el desarrollo de las actividades y servicios turísticos. A nivel mundial se han implementado varias de estas iniciativas, las que pueden ser extrapolables a destinos que desean incursionar en esta variante de realizar turismo (Medina, 2017).

Recientemente, autores como Domínguez et al. (2015), Gillovic et al. (2018), Edgell (2019) y Gillovic y McIntosch (2020), hacen énfasis en la importancia de realizar estudios sobre las acciones que se deben llevar a cabo en los destinos para el diseño y gestión de productos cada vez más accesibles para todos los segmentos de demanda.

En el proceso de evolución del turismo accesible se ha observado una tendencia creciente de esta actividad en países de América Latina y el Caribe (Tite et al., 2021). Precisamente, dentro del área del Caribe, según Salinas et al. (2019), Cuba es uno de los destinos de mayor crecimiento en la actualidad en el Caribe y no se encuentra ajena a la necesidad de satisfacer a este nicho de demanda. Por ello, ha incluido dentro de sus prioridades la atención a este grupo de personas, el cual 
constituye un segmento con gran potencial de desarrollo, permitiendo consigo un aumento del número de visitantes y un incremento del posicionamiento del turismo cubano en el mercado internacional.

La Feria Internacional de Turismo 2019 celebrada en Cuba, fue un escenario propicio para accionar sobre este objetivo. Varios especialistas de la Red Española de Turismo Accesible y de la Red Iberoamericana de Turismo Accesible, de conjunto con especialistas de la agencia de viajes Ecotur y el turoperador Skedio Travel, enunciaron la importancia del desarrollo de esta forma de realizar turismo en el destino y la necesidad de contar con la infraestructura necesaria para todas las actividades y servicios turísticos (Travel Trade Caribbean, 2019).

En Cuba existen, además, Organizaciones No Gubernamentales como la Asociación Nacional de Ciegos y Débiles Visuales (en lo adelante ANCI), la Asociación Nacional de Sordos de Cuba (en lo adelante ANSOC) y la Asociación Cubana de Limitados Físico - Motores (en lo adelante ACLIFIM), las cuales fueron creadas con el fin de atender e insertar a esta gran familia en la vida cotidiana. Dichas organizaciones, además de representar un fuerte apoyo al correcto desarrollo de la sociedad cubana en su conjunto, se encuentran estrechamente vinculadas al sector turístico, ya que son una gran fuente de conocimientos y experiencias en cuanto al trato con personas con alguna limitación especial.

Según los especialistas entrevistados, del número total de visitantes con alguna discapacidad que se recibe en Cuba, el 75\% de los que componen este segmento se corresponden con los que llegan a los destinos de La Habana y Varadero. Estas ciudades cuentan con un considerable número de hoteles para la recepción de visitantes incluyendo turistas con discapacidad (Carmona e Izquierdo, 2019).

Según los análisis realizados por Izaguirre y Torres (2017), Martínez y Boujrouf (2018), Sanz (2018), Guerrero (2018), Tite (2019), Guamán et al. (2019), Porto y Rucci (2019), Sánchez et al. (2020), es sumamente importante que los gestores del turismo, sobre todo en las instalaciones que ofrecen servicios de alojamiento realicen estudios de sus niveles de accesibilidad. A pesar de ello, en los destinos de La Habana y Varadero influye en la atención especializada a este segmento de 
demanda, el nivel de desconocimiento actual que poseen los especialistas de las organizaciones vinculadas a su atención, los cuales refieren desconocer sobre las normativas que regulan la accesibilidad de los hoteles en Cuba.

De igual forma, hasta el momento son escasos los estudios desarrollados sobre la accesibilidad de los establecimientos hoteleros ubicados en La Habana y Varadero, y es tarea priorizada de la agencia de viajes Ecotur como parte de su plan de desarrollo hacia el año 2030, siendo este de suma importancia, ya que a través del mismo se podrá conocer la situación y condición actual del turismo accesible en dichos hoteles, que permitan al turista con discapacidad o diversidad funcional, acceder a un producto turístico en un entorno y una comunicación universal accesible a sus necesidades, con el fin de poder disfrutar del mismo de forma autónoma, cómoda y segura. Además, esta información resultará importante para la toma de decisiones en proyectos de desarrollo del turismo.

Por todo ello, la presente investigación se desarrolló con el objetivo de proponer acciones para elevar el nivel de accesibilidad de los hoteles comercializados por Ecotur en los destinos de La Habana y Varadero.

\section{Metodología}

La investigación, de acuerdo con Hernández et al. (2014), se clasifica como descriptiva. Además, es clasificada como de naturaleza cuantitativa y cualitativa, pues se utilizan también análisis matemáticos y descripciones cualitativas.

Para el desarrollo de la misma, fue necesario la evaluación del nivel de accesibilidad de los hoteles objeto de estudio. Para definir los elementos evaluados por los expertos se tuvo en cuenta los estudios realizados por Fernández (2007), Martos (2012), Suriá y Escalona (2014), Rodríguez (2017) y Guamán et al. (2019). Luego de establecer una comparación entre estas metodologías se tomó como referencia la que incluye un número mayor de ítems a evaluar, Rodríguez (2017). Posteriormente, mediante trabajo de conjunto entre los autores, se elaboró un modelo o instrumento de evaluación adaptado al contexto de la investigación. En este modelo se reagruparon los emplazamientos valorados en dicha investigación y se tuvo en cuenta, además, la existencia de 
personal en el hotel con formación en la atención de personas con alguna discapacidad y la recepción habitual de clientes con discapacidades.

A partir del análisis realizado en conjunto con los expertos, la investigación se desarrolló en cuatro fases (Tabla 1) y la metodología propuesta en el presente estudio fue validada según Juicio de Expertos, a través del Método Delphi.

\section{Tabla 1}

Fases para el desarrollo de la investigación

\begin{tabular}{|c|c|c|}
\hline Fase & Objetivos & $\begin{array}{l}\text { Métodos, técnicas y } \\
\text { herramientas }\end{array}$ \\
\hline $\begin{array}{l}\text { Selección de los } \\
\text { hoteles e } \\
\text { identificación por } \\
\text { categoría y cadena } \\
\text { hotelera }\end{array}$ & $\begin{array}{l}\text { Identificar los hoteles objeto de estudio de la } \\
\text { investigación. } \\
\text { Agrupar, los hoteles, atendiendo a su categoría } \\
\text { y la cadena de gestión a la que pertenecen. } \\
\text { Identificación, en los hoteles seleccionados, los } \\
\text { Departamentos encargados de ofrecer } \\
\text { información directa para la investigación. }\end{array}$ & $\begin{array}{l}\text { Revisión } \\
\text { bibliográfica } \\
\text { Observación directa }\end{array}$ \\
\hline $\begin{array}{l}\text { Evaluación del nivel } \\
\text { de accesibilidad de } \\
\text { las entidades }\end{array}$ & $\begin{array}{l}\text { Diseño del método de obtención de información } \\
\text { a utilizar. } \\
\text { Evaluar el nivel de accesibilidad de las } \\
\text { entidades. }\end{array}$ & $\begin{array}{l}\text { Revisión } \\
\text { bibliográfica } \\
\text { Encuestas } \\
\text { Entrevistas }\end{array}$ \\
\hline $\begin{array}{l}\text { Análisis de la } \\
\text { evaluación general }\end{array}$ & $\begin{array}{l}\text { Identificar el nivel de accesibilidad de los } \\
\text { hoteles evaluados. }\end{array}$ & Análisis estadístico \\
\hline $\begin{array}{l}\text { Propuesta de } \\
\text { acciones }\end{array}$ & $\begin{array}{l}\text { Proponer acciones de mejora para la } \\
\text { accesibilidad en los establecimientos evaluados. }\end{array}$ & Tormenta de ideas \\
\hline
\end{tabular}

Fuente: elaboración propia a partir de lo expuesto por Rodríguez (2017).

\section{Resultados}

\section{Fase 1: selección de los hoteles e identificación por categoría y cadena hotelera}

Los datos muestran que el 20,7\% de los 29 establecimientos (Tabla 2), que formaron parte del estudio, pertenecen a la Cadena hotelera Iberostar con seis hoteles, tres de ellos ubicados en La Habana y tres en Varadero, cuatro de ellos tienen la máxima categoría. Le siguió la cadena Meliá, la cual estuvo representada por cinco hoteles $(17,2 \%)$, todos localizados en Varadero y tres de ellos 
ostentan la categoría cinco estrellas. Del total de instalaciones objeto de estudio, 17 ostentan la categoría cuatro estrellas.

\section{Tabla 2}

Identificación de los hoteles según cadena hotelera y categoría

\begin{tabular}{|c|c|c|c|c|}
\hline $\begin{array}{l}\text { Cadena } \\
\text { Hotelera }\end{array}$ & Habana/Categoría & Varadero/Categoría & $\mathrm{N}^{\mathrm{o}}$ & $\begin{array}{l}\text { Por ciento } \\
\quad(\%)\end{array}$ \\
\hline \multirow{3}{*}{ Iberostar } & Riviera**** & Taíno**** & \multirow{3}{*}{6} & \multirow{3}{*}{20,7} \\
\hline & Parque Central***** & Bella Vista***** & & \\
\hline & $\begin{array}{l}\text { Gran Hotel } \\
\text { Packard***** }\end{array}$ & Laguna Azul***** & & \\
\hline \multirow{5}{*}{ Meliá } & \multirow[t]{5}{*}{ 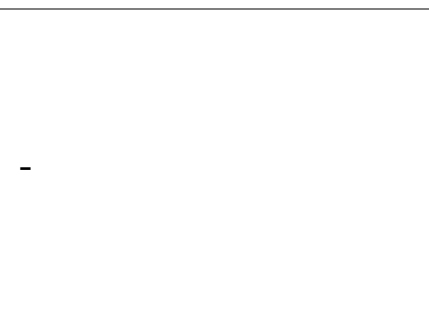 } & Paradisus $* * * * *$ & \multirow{5}{*}{5} & \multirow{5}{*}{17,2} \\
\hline & & Princesa del Mar***** & & \\
\hline & & Meliá Internacional $* * * * *$ & & \\
\hline & & Marina Varadero $* * * *$ & & \\
\hline & & $\begin{array}{l}\text { Meliá Península } \\
\text { Varadero**** }\end{array}$ & & \\
\hline \multirow{3}{*}{ Blue Diamond } & \multirow{3}{*}{$\begin{array}{l}\text { Memories Miramar } \\
\text { Havana**** }\end{array}$} & Memories Varadero**** & \multirow{3}{*}{4} & \multirow{3}{*}{13,8} \\
\hline & & $\begin{array}{l}\text { Grand Memories } \\
\text { Varadero**** }\end{array}$ & & \\
\hline & & Royalton Hicacos $* * * *$ & & \\
\hline \multirow{2}{*}{ H10 } & \multirow{2}{*}{ Panorama**** } & Ocean Patriarca***** & \multirow{2}{*}{3} & \multirow{2}{*}{10,3} \\
\hline & & Ocean Vista Azul***** & & \\
\hline \multirow{2}{*}{$\mathrm{ROC}$} & \multirow{2}{*}{ Presidente**** } & Arenas Doradas $* * * *$ & \multirow{2}{*}{3} & \multirow{2}{*}{10,3} \\
\hline & & ROC Barlovento**** & & \\
\hline Be Live & - & Experience Turquesa $* * * *$ & 1 & 3,4 \\
\hline Gran Caribe & Nacional $* * * * *$ & - & 1 & 3,4 \\
\hline $\mathrm{NH}$ & NH Capri-Victoria***** & - & 1 & 3,4 \\
\hline Saratoga & Saratoga $* * * * *$ & - & 1 & 3,4 \\
\hline Kempinski & $\begin{array}{l}\text { Manzana } \\
\text { Kempinski***** }\end{array}$ & - & 1 & 3,4 \\
\hline Grupo Posadas & - & $\begin{array}{l}\text { Fiesta Americana Punta } \\
\text { Varadero**** }\end{array}$ & 1 & 3,4 \\
\hline Labranda & - & Labranda Varadero $* * * *$ & 1 & 3,4 \\
\hline ACCOR & SO Paseo del Prado & - & 1 & 3,4 \\
\hline Total & 11 & 18 & 29 & 100,0 \\
\hline
\end{tabular}

Fuente: elaboración propia. 


\section{Fase 2: evaluación del nivel de accesibilidad de las entidades}

Los resultados de esta fase de la investigación, se detallarán por apartados tal y como los plantea Rodríguez (2017) (Anexo 1). De igual forma, es válido resaltar que se presenta la descripción de los apartados teniendo en cuenta las respuestas a las encuestas realizadas, así como las entrevistas a algunos directivos de las instalaciones objeto de estudio.

\section{Accesibilidad exterior}

Analizando los resultados obtenidos al evaluar los ítems relacionados con este apartado, se obtuvo que el 100\% de los establecimientos hoteleros se encuentran claramente señalizados y el recorrido exterior hasta el acceso no es adecuado en seis hoteles $(20,7 \%)$.

En cuanto a las plazas de aparcamiento para personas con movilidad reducida, el 96,5\% de los hoteles no posee ninguna, por lo tanto, no cumplen con ninguno de los requisitos en lo que respecta a este parámetro, es decir, dimensiones, número y señalización de las mismas, solamente cumple con todos los parámetros, el hotel SO Paseo el Prado de La Habana.

Al comparar estos resultados con investigaciones similares en otros destinos, se puede comprobar que difieren de los descritos, en varias ciudades españolas, por autores como Rodríguez (2017) y Fernández (2007) al observar la existencia de varios hoteles con plazas de aparcamiento para personas con discapacidad y dentro de ellos, algunos que cumplen con la normativa. En Alicante, Suriá y Escalona (2014), plantean dificultades en cuanto a falta de señalética, carencia de rampas de acceso, mala ubicación, falta de ascensores adaptados en el caso de los estacionamientos interiores y poco espacio.

Los resultados obtenidos según accesibilidad exterior, muestran que el 75,9\% de hoteles evaluados, presentan un nivel de accesibilidad bajo.

\section{Acceso principal al recinto}

Al valorar el nivel de accesibilidad en este apartado se observó que, del total de hoteles, 25 (86,2\%) presentan escalones o desniveles para acceder al establecimiento; sin embargo, solo en tres casos 
(10,3\%) (Grand Hotel Packard, NH Capri y Nacional) de La Habana, las escaleras sobrepasan los 12 escalones; en 11 instalaciones solo existe un escalón y cuatro presentan un desnivel.

En el 96,5\% de los hoteles, la escalera de acceso es cómoda y segura o no es necesaria, solo en el hotel Riviera de la capital, se constató que la escalera de acceso al establecimiento no cumple con los requisitos de accesibilidad. Estos resultados no coinciden con los descritos por Suriá y Escalona (2014), quienes evaluaron los diferentes parámetros de accesibilidad a través de encuestas a personas con discapacidad motora, y un porciento de los encuestados señaló que las escaleras son las que más dificultad presentan para acceder a los hoteles de Alicante.

El 89,6\% de los establecimientos dispone de rampa, cómoda y segura, para acceder al hotel o no la necesita, solo los hoteles Riviera, Memories Miramar y Presidente de la Habana, no cuentan con rampa para salvar los escalones o desniveles del acceso. Estos hallazgos superan a los descritos por otros autores como Rodríguez (2017) y Suriá y Escalona (2014) en Almuñécar y Cádiz, respectivamente.

Siete entidades hoteleras $(24,1 \%)$, seis de la Habana (Riviera, Hotel Grand Packard, Nacional, SO Paseo del Prado y Presidente) y uno de Varadero (ROC Barlovento), no cuentan con una plataforma elevadora para personas con movilidad reducida, mientras que el resto de los establecimientos hoteleros no la necesitan. Estos resultados no coinciden con los descritos por Rodríguez (2017), ya que un elevado porcentaje de las instalaciones hoteleras visitadas poseen plataformas elevadoras que cumplen con la norma vigente referida para esa investigación.

En cuanto a la dimensión de la puerta de acceso, el 100\% de los hoteles cumplen con los requisitos de accesibilidad, al ser abierta o con una anchura mayor o igual a $80 \mathrm{~cm}$, resultados similares a los expuestos por Rodríguez (2017) quien señaló como alta la accesibilidad en este aspecto.

Por otro lado, Fernández (2007) mostró los niveles de accesibilidad de los accesos al recinto en Cádiz mediante la evaluación, de manera independiente, de los accesos y las entradas, encontrando niveles de accesibilidad muy altos para varios alojamientos en cuanto a los accesos, mientras que altos para las puertas de entrada en un elevado porcentaje de los mismos. 
En el 96,5\% de los alojamientos objetos de estudio, el mostrador de recepción no tiene doble altura, ni tienen una altura adecuada para ser considerados accesibles para clientes usuarios de silla de ruedas. Solo el hotel Royalton Hicacos de Varadero cumplió con este criterio, al tener el mostrador de recepción a una altura adecuada para personas usuarias de silla de ruedas.

Al comparar estos resultados con los reportados en hoteles evaluados de dos ciudades diferentes de España, se puede plantear que están en correspondencia con los obtenidos por Rodríguez (2017) quien llamó la atención sobre la no disponibilidad de mostradores con dos alturas para hacerlos accesibles y difieren de los obtenidos por Fernández (2007), quien reveló la presencia de hoteles que disponen de un mostrador con las características de accesibilidad requerida.

El análisis anterior muestra que el 65,5\% de hoteles presenta un nivel accesibilidad según acceso principal alto, y en un nivel muy alto cinco hoteles $(17,2 \%)$, que incluyen el Panorama de La Habana y Taíno, Paradisus, Royalton Hicacos y Meliá Península de Varadero.

\section{Comunicación vertical}

Al valorarse si el hotel es de planta única o si las habitaciones adaptadas y servicios comunes se encuentran en la planta de acceso, no se obtuvieron resultados positivos en 17 instalaciones (58,6\%), todos los hoteles de la Habana excepto SO Paseo del Prado y siete de Varadero. El 100\% de los hoteles cuentan con escaleras interiores, que fueron consideradas accesibles al ser cómodas y seguras para personas con discapacidad, según normativa vigente, resultados que son superiores a los reportados por Fernández (2007) y Rodríguez (2017).

En el hotel Labranda Varadero, que representa el 3,4\% del total, se observó que los ascensores no cumplen con las dimensiones adecuadas, único hotel que tampoco su ascensor tiene acceso a las habitaciones adaptadas; no siendo así para los espacios o áreas comunes, donde el número de hoteles aumentó a cinco (17,2\%). Los hoteles que no cumplen con este criterio son el Panorama, NH Capri-Victoria y SO Paseo del Prado de La Habana, así como el Labranda y Meliá Internacional de Varadero. 
En cuanto a la capacidad para una camilla en el ascensor se identificó en el 41,3\% de los alojamientos, el Riviera de La Habana y once localizados en Varadero; en las facilidades para ciegos y sordos, en los ascensores, se observó en $24(82,7 \%)$ y 27 (93,1\%) hoteles respectivamente, detectándose que el Riviera y el Labranda Varadero no tienen facilidades para ninguna de las dos discapacidades, mientras que el Nacional y NH Capri-Victoria de La Habana y el Royalton Hicacos de Varadero solo tiene facilidades para discapacitados auditivos.

Otros autores han evaluado la accesibilidad de los ascensores en establecimientos hoteleros y han obtenido resultados menos favorables (Fernández, 2007; Suriá y Escalona, 2014). Por otro lado, Rodríguez (2017), percibió mejores resultados, los cuales coinciden con los encontrados en la presente investigación.

Según comunicación vertical, el 55,2\% de los hoteles obtuvo un nivel alto de accesibilidad, mientras que el Labranda Varadero $(3,4 \%)$ presenta un nivel muy bajo y el NH Capri-Victoria de La Habana $(3,4 \%)$ un nivel medio.

\section{Comunicación sensorial}

Se comprobó que $100 \%$ de los hoteles incluidos en la investigación cumplieron con el criterio de iluminación natural y artificial adecuada, así como con la señalización escrita y gráfica para indicar las diferentes estancias de los establecimientos. Resultados coincidentes en cuanto a la iluminación y no coincidentes en cuanto a la señalización, fueron reportados por Rodríguez (2017).

El 100\% de los hoteles objeto de estudio, obtuvo resultados negativos en cuanto a la información del establecimiento y sus servicios para personas ciegas, existencia de ayudas técnicas para personas sordas, personal en el establecimiento con conocimiento de lenguaje de signos y servicio de WIFI Free en las diferentes áreas de las entidades. Estos resultados conllevaron a que todos los hoteles obtuvieran la misma puntuación en este apartado y por tanto todos los alojamientos se encuentran en el nivel bajo de accesibilidad en lo que a comunicación sensorial se refiere, lo cual está no está en correspondencia con el nivel medio obtenido en este emplazamiento por Rodríguez (2017).

\section{Áreas Comunes}


Las puntuaciones obtenidas en el apartado Áreas Comunes, se obtuvieron mediante la evaluación de seis subapartados. El primer subapartado fue diseñado para la evaluación de las zonas de paso o recorridos. Se encontró que solo el hotel Taíno de Varadero $(3,4 \%)$ no tiene escalones o desniveles aislados en zonas o recorridos del hotel.

De los 28 establecimientos con escalones o desniveles en plantas o recorridos del hotel, 11 (37,9\%) no disponen de rampas o plataformas elevadoras para salvar los escalones o desniveles detectados y en $24(82,7 \%)$ existen espacios o áreas comunes sin acceso. El estudio realizado por Suriá y Escalona (2014) demuestra que en otros hoteles también existen dificultades para acceder a las diferencias estancias intrahoteleras como la recepción, el Bar/Restaurante e incluso para acceder las habitaciones.

Se constató que el 100\% de las instalaciones hoteleras cuenta con pasillos que permiten el cruce de dos personas simultáneamente. En el caso particular del Hotel Manzana Kempinski, se consideró darle la puntuación, por solo identificarse el pasillo hacia el gimnasio sin las dimensiones adecuadas.

Se encontró pavimento deslizante en algunas estancias de 24 hoteles $(82,7 \%)$, diez de la ciudad de la Habana y 14 de Varadero. Estos resultados difieren de lo reportado por Rodríguez (2017), ya que, en un porcentaje bajo de las instalaciones visitadas, el pavimento no es antiresbalante.

Rodríguez (2017) evaluó los ámbitos de paso y los pavimentos, de manera independiente, ambos parámetros formaron parte de la evaluación de la circulación horizontal en los recintos. Puntualizó que un alto porcentaje de los hoteles incluidos en el estudio, cumplen con los criterios valorados en cuanto a los ámbitos de paso y el pavimento respectivamente, lo cual no coincide con los resultados obtenidos en los hoteles objeto de estudio, donde se encontró un 38,6\% de accesibilidad de manera general en cuanto a las zonas de paso y recorridos del hotel, lo que se traduce en un nivel bajo de accesibilidad en este subapartado.

El segundo subapartado se concibió para evaluar las puertas en las diferentes entidades, encontrándose que en el $100 \%$ de los hoteles, las puertas tienen un ancho mínimo de $80 \mathrm{~cm}$ y las 
mismas presentan placas informativas, a excepción del Meliá Internacional de Varadero. Sin embargo, estas placas informativas solo se encuentran señalizadas en braille o alto relieve en el $24,1 \%$ de los hoteles, cuatro ubicados en La Habana y tres en Varadero. En este apartado se obtuvo un $74,6 \%$ de accesibilidad (nivel alto), lo cual difiere del porcentaje superior alcanzado en Almuñécar por Rodríguez (2017).

El tercer subapartado está relacionado con la disponibilidad en los establecimientos de baño común adaptado. El 82,7\% de las instalaciones disponen de baño adaptado en las zonas comunes, solo los hoteles Riviera, Nacional, Memories y Presidente de La Habana y Grand Memories de Varadero, no pueden ofrecer a los clientes con discapacidad, baños adaptados en áreas comunes. En el caso del Grand Memories, el baño existe, pero se encuentra cerrado por reparaciones, por tanto, no obtuvo la puntuación durante la visita. Estas instalaciones, al no disponer de baños aptos para su uso, en las estancias comunes, no cumplen con ninguno de los requisitos evaluados en este subapartado.

En todos los hoteles que cuentan con baños adaptados, estos se encuentran situados en planta baja con recorrido accesible o en plantas accesibles y además cuentan con señalización específica a excepción de los hoteles Experience Turquesa y ROC Barlovento de Varadero. De los 24 hoteles que disponen de baños adaptados en zonas comunes, tanto las dimensiones del baño como de su puerta para personas usuarias de sillas de ruedas, se encontraron adecuadas en 22 instancias, encontrándose que el hotel NH Capri-Victoria y Saratoga de La Habana no cumplen con las dimensiones establecidas. En cuanto a las ayudas técnicas, en nueve hoteles, los baños no disponen de todas las requeridas según la normativa vigente, el Hotel Parque Central de La Habana y los hoteles Taíno, Laguna Azul, Marina Varadero, Experience Turquesa, ROC Barlovento, Fiesta Americana, Labranda y Meliá Península de Varadero.

En este subapartado se obtuvo un grado de accesibilidad alto (70,8\%), existiendo diferencias entre los resultados encontrados en la presente investigación y los reportados por Fernández (2007) en hoteles de Cádiz. En Almuñécar, los resultados concuerdan con los obtenidos en los hoteles de La Habana y Varadero (Rodríguez, 2017). 
El cuarto subapartado se refiere a la disponibilidad de Cafetería/Restaurante en los establecimientos hoteleros, así como sus características de accesibilidad. Se observó que el 100\% de los alojamientos disponen de Cafeterías y Restaurantes con mesas con alturas entre 75 y $80 \mathrm{~cm}$ y ámbitos de paso principales que permiten el cruce de dos personas simultáneamente. No obstante, ninguno cuenta con barras de dos alturas diferentes en las Cafeterías.

Se comprobó que solo los hoteles Manzana Kempinski de La Habana y Laguna Azul de Varadero disponen de menús específicos para celíacos o alérgicos. En el resto de los hoteles, los clientes con necesidades específicas las hacen saber en la reserva y esa información es colegiada con el resto del staff para darle un servicio especial de acuerdo a los requerimientos específicos de cada cliente, además en el buffet presentan un área con alimentos clasificados.

De forma general se encontró en este subapartado un nivel de accesibilidad alto (61,5\%). Estos resultados concuerdan en gran medida con los reportados por Rodríguez (2017) y Suriá y Escalona (2014); sin embargo, llama la atención la no disponibilidad de barras con dos alturas para hacer las Cafeterías de los hoteles, de la Habana y Varadero, accesibles.

El quinto subapartado evalúa la accesibilidad de la piscina. No se consideró darle la puntuación a ninguno de los hoteles objeto de estudio, ya que a pesar de que algunos cuentan con piscina nivel cero, en ninguna instalación visitada se ofrece a los clientes la posibilidad de disfrutar de manera cómoda y segura de la piscina, resultados que están muy por debajo de los obtenidos por otros investigadores (Rodríguez, 2017; Suriá y Escalona, 2014).

Este subapartado obtuvo un nivel muy bajo de accesibilidad. Es importante ser conscientes de que, para los clientes, como afirman Suriá y Escalona (2014), "bien por su recreo o bien por sus propiedades (...) uno de los bienes más preciados de un hotel es la piscina y por ello, es muy necesario que esté adaptada y con buen acceso".

El sexto subapartado se refiere a la protección contra incendio y emergencias. Se confirmó que el $100 \%$ de los alojamientos cuentan con un plan de emergencia para la evacuación de personas con diferentes tipos de discapacidad. Sin embargo, se identificó que los hoteles Panorama y Presidente 
de La Habana y Princesa del Mar y Royalton Hicacos de Varadero (13,8\%), no disponen de los dos tipos de alarma requeridas (sonora y luminosa).

Los resultados obtenidos fueron ligeramente más favorables que los presentados por Rodríguez (2017) quien encontró un bajo número de instalaciones que no cumplen la norma en cuanto a sistemas de alarma y de evacuación en caso de incendio. En este subapartado se obtuvo un nivel muy alto de accesibilidad $(92,7 \%)$.

Prevaleció el nivel alto de accesibilidad en las zonas comunes con el 69,0\% de los casos y siete establecimientos $(24,1 \%)$ obtuvieron el nivel medio, sin embargo, el nivel de accesibilidad general obtenido en este subapartado fue medio con un $60,1 \%$.

\section{Habitaciones}

El estudio permitió conocer que la disponibilidad de habitaciones adaptadas es del 100\% de los establecimientos hoteleros objeto de estudio. No obstante, ninguno de los hoteles cumple con la normativa cubana e internacional de disponer de 1 habitación accesible por cada 20 habitaciones corrientes. Estos resultados superan a los reportados en Cádiz; sin embargo, en esta ciudad se reportan hoteles con un elevado número de habitaciones adaptadas (45, 77, 90, 100 y 126) (Fernández, 2007).

El máximo número de habitaciones adaptadas disponibles en los hoteles visitados en La Habana y Varadero, fue de 14 habitaciones en el hotel Memories Varadero, aunque en correspondencia con el número total de habitaciones representa el 1,3\%, mientras que el hotel Saratoga de La Habana, a pesar de contar con solo 96 habitaciones, cuenta con dos adaptadas representando el 2,1\%.

En el presente estudio se percibió que las habitaciones adaptadas y su puerta son mayores que los estándar, en el 58,6\% de las instalaciones, seis de la Habana y 11 de Varadero. El 96,5\% de las estancias disponen de baño adaptado en las habitaciones accesibles, encontrándose que solo el hotel ROC Presidente no cumple con este criterio. No obstante, todos los baños no cumplen con los requisitos de accesibilidad medidos en la investigación, encontrándose siete hoteles $(24,1 \%)$, seis en La Habana y uno en Varadero, donde las dimensiones del baño y su puerta no son adecuadas 
para una persona usuaria de silla de ruedas; además, en seis instalaciones $(20,7 \%)$, los baños adaptados no disponen de las ayudas técnicas requeridas para personas con discapacidad.

Se observó disponibilidad de duchas en todas las habitaciones adaptadas. No obstante, en cuatro hoteles las duchas no cumplen con los requisitos normados para personas usuarias de sillas de ruedas, el Riviera de La Habana y Royalton Hicacos, Experience Turquesa y Fiesta Americana de Varadero.

Se identificaron instalaciones domóticas en las habitaciones adaptadas de los hoteles SO Paseo del Prado de La Habana y Meliá Internacional de Varadero, representando el 11,6\% del total. En cuanto a la señalización de estas habitaciones, solo cuatro establecimientos hoteleros ubicados en la capital no cuentan con puertas bien señalizadas (Parque Central, Nacional, NH Capri-Victoria y Presidente), representando el $13,7 \%$ de los casos y en solo dos alojamientos (6,8\%), estas señalizaciones se encuentran en braille o alto relieve (Memories Miramar de La Habana y Fiesta Americana de Varadero).

Los resultados obtenidos permiten mostrar el nivel de accesibilidad en cuanto a habitaciones adaptadas. De forma general se observó un nivel alto de accesibilidad alto en este apartado (61,4\%), obteniéndose este nivel en el 72,5\% de las estancias hoteleras. En la capital dos hoteles obtuvieron resultados muy distantes en cuanto a habitaciones se refiere, el ROC Presidente obtuvo un nivel de accesibilidad muy bajo, mientras que el SO Paseo del Prado obtuvo un nivel muy alto de accesibilidad representando cada uno el 3,4\% de la muestra.

\section{Formación}

En este apartado el estudio de campo realizado arrojó que el 93,1\% de los hoteles no cuentan con personal capacitado en la atención de clientes con algún tipo de discapacidad, solo los hoteles SO Paseo del Prado de la Habana y ROC Barlovento de Varadero, cumplen con este requisito, por tanto, predominó el nivel muy bajo en este apartado al obtenerse un 7,4\% de accesibilidad. 
La falta de capacitación del personal pudiera ser consecuencia de que el turismo accesible en Cuba está comenzando a promoverse y desarrollarse. No obstante, los resultados observados son similares a los expuestos por autores como Suriá y Escalona (2014), quienes indicaron que una de las mayores dificultades a las que se enfrentan las personas con discapacidad en los hoteles de Alicante, es la falta de capacitación del personal para atenderlos y Guamán et al. (2019) quienes encontraron una brecha, en un elevado porcentaje de los establecimientos evaluados en Ambato, Ecuador, entre las especificaciones de calidad y el servicio realmente ofrecido, mostrando que una de las causas es no contar con el personal adecuado para cumplir con las especificaciones del cliente.

Llama la atención que la formación del personal es deficiente o nula, a pesar de tratarse de hoteles situados en países con economía y nivel de desarrollo diferentes como son España, Ecuador y Cuba.

\section{Clientes}

Según las entrevistas a directivos de los diferentes establecimientos, el 93,1\% reciben habitualmente este tipo de clientes obteniéndose un nivel muy alto en este apartado. Dos hoteles ubicados en Varadero, el Fiesta Americana y el Labranda Varadero, no son visitados de manera habitual por personas con discapacidad. A pesar de la falta de capacitación del personal de los hoteles en la atención a este sector de la población, constatada en el apartado anterior, llama la atención que personas con algún tipo de discapacidad, opten por estos destinos turísticos, lo que habla a favor de la calidad y el buen trato que se brinda a los huéspedes en estas instalaciones.

\section{Fase 3: análisis de la evaluación general}

\section{Nivel de accesibilidad global por apartados}

Con el objetivo de conocer los parámetros que influyen en mayor o menor medida en el nivel de accesibilidad hotelera alcanzado, se determinó la accesibilidad global por apartados (Figura 1). Además, se compararon los resultados entre ambas ciudades para conocer si existen diferencias entre los factores que influyen en los hoteles de ciudad y de playa. 


\section{Figura 1}

Nivel de accesibilidad global por apartados

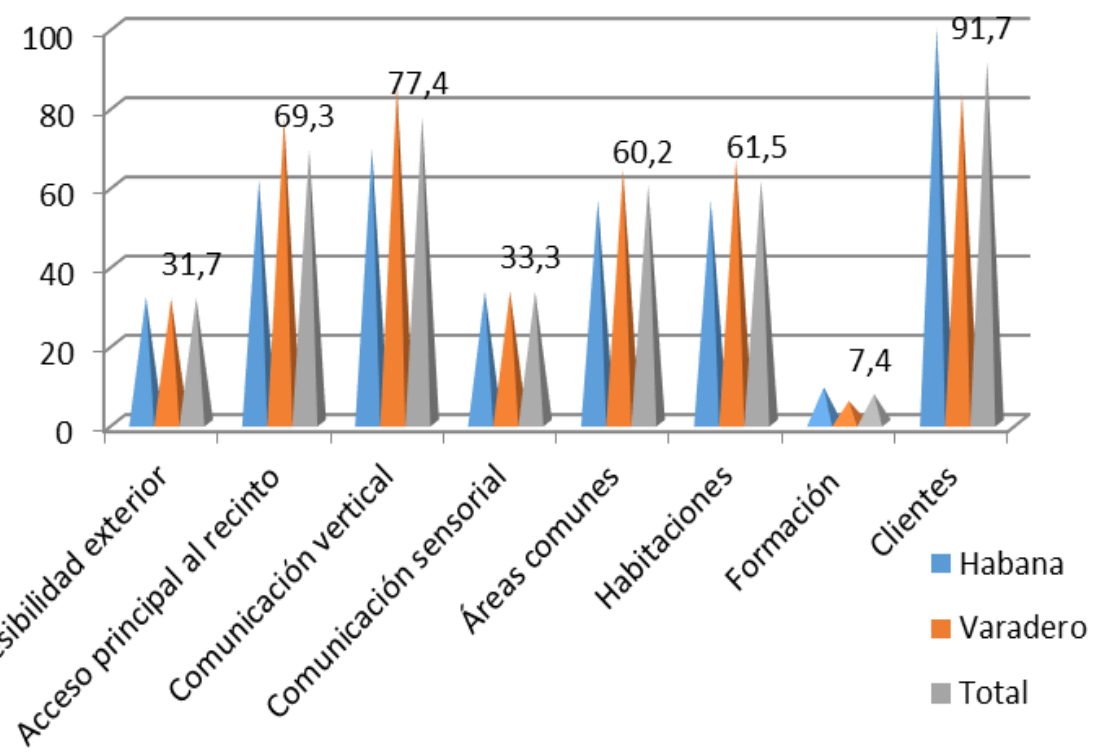

Fuente: elaboración propia.

El apartado que obtuvo el menor porcentaje $(7,4 \%)$ fue la formación de los trabajadores del hotel en la atención a personas con discapacidad, quedando en un nivel muy bajo de accesibilidad. Este parámetro pudiera mejorarse sin afectaciones mayores al presupuesto de las entidades hoteleras, ya que para promover la capacitación del personal no se requieren cambios en la infraestructura.

La accesibilidad exterior y la comunicación sensorial (31,7\% y 33,3\%, respectivamente), también influyeron negativamente, al obtener un nivel bajo de accesibilidad. En cuanto a la accesibilidad exterior no se encontraron diferencias entre los hoteles localizados en La Habana y los de Varadero, los cuales alcanzaron 31,8\% y 31,5\% de accesibilidad respectivamente en este apartado. En ambas ciudades influyó la no disponibilidad de plazas reservadas en los hoteles para personas con discapacidad.

Cada ciudad aportó un 33,3\% de accesibilidad en cuanto a comunicación sensorial al no disponerse en ninguno de los hoteles, de información para personas ciegas, de ayudas técnicas para personas sordas, personal con conocimiento del lenguaje de signos, ni servicio de WIFI Free en las diferentes estancias de los hoteles. 
El apartado más accesible fue la recepción habitual de clientes con alguna discapacidad (91,7\%), que se tuvo en cuenta para conocer en cierta medida la disposición de este segmento de la población de alojarse en los establecimientos visitados. Le siguió la comunicación vertical (77,4\%) y el acceso principal al recinto $(69,3 \%)$.

Estos resultados no coinciden con los expuestos por Rodríguez (2017), quien en su investigación también comparó el nivel de accesibilidad por emplazamientos evaluados y concluyó que, en los hoteles visitados en Almuñécar, los establecimientos hosteleros como restaurantes, cafeterías y bares fueron los más accesibles, mientras que el menos accesible fue la señalización. Los niveles de accesibilidad obtenidos en cada uno de los apartados evaluados, se comportaron de manera similar en ambas ciudades.

\section{Nivel de accesibilidad global}

En la Tabla 3 se muestran los resultados obtenidos por cada hotel según apartados analizados. Este análisis permitió identificar que en la formación del personal fue donde mayores deficiencias se detectaron, seguidas de la accesibilidad exterior y la comunicación sensorial. El hotel que posee mayor grado de accesibilidad es el SO Paseo del Prado de la capital $(72,1 \%)$, de la cadena ACCOR, con un nivel alto, el cual ostenta categoría 5 estrellas, mientras que el de menor nivel es el hotel ROC Presidente de La Habana (37,7\%), único hotel que obtuvo un nivel bajo de accesibilidad. 
Tabla 3

Nivel de accesibilidad hotelera

\begin{tabular}{|c|c|c|c|c|c|c|c|c|c|c|}
\hline Hoteles & AExt & APR & $\mathrm{CV}$ & CS & $\mathrm{AC}$ & $\mathrm{H}$ & For & Cli & Total & $\%$ \\
\hline \multicolumn{11}{|c|}{ HABANA } \\
\hline Riviera & 2 & 3 & 5 & 2 & 8 & 5 & 0 & 1 & 26 & 42,6 \\
\hline Parque Central & 2 & 6 & 6 & 2 & 12 & 7 & 0 & 1 & 36 & 59,0 \\
\hline Gran Hotel Packard & 1 & 4 & 6 & 2 & 14 & 8 & 0 & 1 & 36 & 59,0 \\
\hline Nacional & 2 & 4 & 5 & 2 & 10 & 5 & 0 & 1 & 29 & 47,5 \\
\hline Panorama & 2 & 7 & 5 & 2 & 13 & 8 & 0 & 1 & 38 & 62,3 \\
\hline Memories Miramar & 1 & 5 & 6 & 2 & 12 & 6 & 0 & 1 & 33 & 54,1 \\
\hline NH Capri-Victoria & 2 & 4 & 4 & 2 & 12 & 5 & 0 & 1 & 30 & 49,2 \\
\hline Saratoga & 1 & 6 & 6 & 2 & 13 & 5 & 0 & 1 & 34 & 55,7 \\
\hline Presidente & 1 & 4 & 6 & 2 & 7 & 2 & 0 & 1 & 23 & 37,7 \\
\hline SO Paseo del Prado & 5 & 5 & 6 & 2 & 15 & 9 & 1 & 1 & 44 & 72,1 \\
\hline Manzana Kempinski & 2 & 6 & 6 & 2 & 16 & 8 & 0 & 1 & 41 & 67,2 \\
\hline \multicolumn{11}{|c|}{ VARADERO } \\
\hline Taíno & 2 & 7 & 6 & 2 & 16 & 8 & 0 & 1 & 42 & 68,9 \\
\hline Bella Vista & 2 & 6 & 7 & 2 & 14 & 7 & 0 & 1 & 39 & 63,9 \\
\hline Laguna Azul & 2 & 6 & 6 & 2 & 14 & 8 & 0 & 1 & 39 & 63,9 \\
\hline Ocean Patriarca & 2 & 6 & 8 & 2 & 14 & 8 & 0 & 1 & 41 & 67,2 \\
\hline Ocean Vista Azul & 2 & 6 & 6 & 2 & 13 & 7 & 0 & 1 & 37 & 60,7 \\
\hline Paradisus & 2 & 7 & 8 & 2 & 15 & 8 & 0 & 1 & 43 & 70,5 \\
\hline Princesa del Mar & 1 & 6 & 8 & 2 & 15 & 8 & 0 & 1 & 41 & 67,2 \\
\hline Marina Varadero & 2 & 6 & 6 & 2 & 13 & 7 & 0 & 1 & 37 & 60,7 \\
\hline Grand Memories & 2 & 6 & 8 & 2 & 9 & 7 & 0 & 1 & 35 & 57,4 \\
\hline Royalton Hicacos & 2 & 7 & 6 & 2 & 13 & 7 & 0 & 1 & 38 & 62,3 \\
\hline Experience Turquesa & 2 & 6 & 8 & 2 & 12 & 7 & 0 & 1 & 38 & 62,3 \\
\hline Arenas Doradas & 2 & 6 & 8 & 2 & 15 & 7 & 0 & 1 & 41 & 67,2 \\
\hline Roc Barlovento & 2 & 5 & 8 & 2 & 14 & 7 & 1 & 1 & 40 & 65,6 \\
\hline Fiesta Americana & 2 & 6 & 8 & 2 & 14 & 7 & 0 & 0 & 39 & 63,9 \\
\hline Labranda Varadero & 1 & 6 & 1 & 2 & 12 & 8 & 0 & 0 & 30 & 49,2 \\
\hline Memories Varadero & 2 & 6 & 8 & 2 & 13 & 7 & 0 & 1 & 39 & 63,9 \\
\hline Meliá Internacional & 2 & 7 & 5 & 2 & 13 & 8 & 0 & 1 & 38 & 62,3 \\
\hline Meliá Península & 2 & 6 & 8 & 2 & 13 & 6 & 0 & 0 & 37 & 60,7 \\
\hline
\end{tabular}

Leyenda: A Ext: Accesibilidad exterior, APR: Acceso principal al recinto, CV: Comunicación vertical, CS: Comunicación sensorial, AC: Áreas comunes, H: Habitaciones, For: Formación, Cli: Clientes.

Fuente: elaboración propia. 


\section{Fase 4: propuesta de acciones}

A partir del análisis realizado, se proponen acciones preliminares en aras de mejorar la accesibilidad en los establecimientos hoteleros objeto de estudio:

\section{General}

1. Implementar en cada instalación hotelera un sistema de evaluación de accesibilidad, para tener claramente identificadas aquellas dependencias en las que es preciso mejorar la accesibilidad, eliminando las barreras físicas existentes.

A partir de aquí, habría que establecer un plan de actuación para lograr la accesibilidad hotelera en la totalidad o mayoría de los establecimientos hoteleros.

\section{Formación del Personal}

1. Elaborar un plan de capacitación para la atención de personas con discapacidad en todas las estancias hoteleras.

2. Extender a todos los establecimientos hoteleros tanto de la Habana y Varadero, como del resto del país el estudio sobre la evaluación de cada hotel en términos de accesibilidad.

3. Desarrollar cursos de capacitación sobre el lenguaje de signos para los trabajadores de las instalaciones.

4. Realizar cursos, seminarios y congresos en las diferentes entidades, con el fin de preparar a todo el personal del sector del turismo sobre el desarrollo del turismo accesible, así como las necesidades y expectativas de este segmento de demanda.

5. Establecer convenios con organizaciones como la ANCI, ANSOC o ACLIFIM que le permita al personal del hotel conocer las principales características de las personas con alguna necesidad especial.

\section{Accesibilidad Exterior}

1. Incorporar plazas de aparcamiento, que cumplan con la normativa requerida, para personas con movilidad reducida. 


\section{Comunicación Sensorial}

1. Incorporar a las entidades, señaléticas en braille o alto relieve para personas ciegas o débiles visuales.

2. Añadir el servicio de WIFI Free en todas las áreas del hotel, permitiendo una mejor comunicación entre los clientes, en especial aquellos que posean limitaciones auditivas.

3. Incorporar el servicio de ayudas técnicas (teléfono de texto, fax, bucles magnéticos, despertador vibrador, etc.) en las diferentes áreas de los hoteles.

\section{Acceso Principal al Recinto}

1. Eliminar barreras físicas como escalones, muros o desniveles.

2. Modificar los mostradores de las recepciones, así como las barras de las cafeterías y bares para que puedan ser utilizadas por personas usuarias de sillas de ruedas.

El resto de las acciones deben estar enfocadas a eliminar las brechas existentes en el resto de los apartados, pues a pesar de presentar un nivel alto, se debe lograr establecer estándares que permitan alcanzar un nivel muy alto de accesibilidad ( $\geq 81 \%$ ).

\section{Conclusiones}

Con el desarrollo de la presente investigación se puede concluir que el desarrollo del turismo accesible se ha ampliado, no sólo a las personas con discapacidad, sino a todos los individuos, independientemente de sus condiciones, ya que no sólo las personas con alguna discapacidad se benefician de la accesibilidad en el turismo.

Por su parte, en Cuba, se carece de estudios de los niveles de accesibilidad de las entidades de alojamiento, lo que dificulta la posibilidad de brindar servicios de calidad a este segmento de demanda. Teniendo en cuenta la importancia del tema se hace imperante desarrollar diagnósticos de accesibilidad en todos los destinos del país.

Al evaluar los niveles de accesibilidad en los hoteles objeto de estudio, se determinaron varias insuficiencias que evidencian la necesidad de continuar en la mejora de las infraestructuras que 
permitan elevar la calidad de los servicios. La investigación permitió determinar que la formación del personal posee un nivel de desarrollo muy bajo, mientras que la comunicación sensorial y la accesibilidad exterior obtuvieron un nivel bajo, influyendo negativamente la falta de ayudas técnicas y de carácter humano para personas con discapacidad, así como plazas reservadas para personas con discapacidad en los aparcamientos.

Se puede afirmar además que, ninguno de los hoteles objeto de estudio puede ser considerado completamente accesible, ya que no alcanzan un grado de accesibilidad del $100 \%$.

En la última fase, se proponen un total de 12 acciones preliminares para elevar los índices de accesibilidad, las cuales constituyen el punto de partida para que las entidades diseñen planes de acción en pos de elevar los niveles de accesibilidad de sus instalaciones.

Por tanto, la investigación constituye una guía para que los gestores del turismo en Cuba puedan desarrollar estos estudios de diagnóstico como primer paso para crear un destino totalmente inclusivo.

\section{Referencias}

(1) Banco Mundial. (2019). Discapacidad. https://www.bancomundial.org/es/topic/disability\#1

(2) Bernaza, A. (2019). Diseño de propuesta de un producto turístico accesible para la Agencia de Viajes San Cristóbal "Sentir la primera Plaza” [Tesis de pregrado, Universidad de La Habana].

(3) Buhalis, D., \& Darcy, S. (2011). Accessible Tourism. Concepts and Issues. Channel View Publications.

(4) Carmona, E., \& Izquierdo, L. (2019). ¿El turismo impulsa el desarrollo de otros sectores en Cuba? [Podcast]. http://www.cubadebate.cu/especiales/2019/06/18/el-turismo-impulsa-eldesarrollo-de-otros-sectores-en-cuba-podcast/

(5) Clemente, J. A. (2015). El Turismo Social Accesible: Un análisis sociológico en la Región de Murcia [Tesis de doctorado, Universidad de Murcia]. 
(6) Cruces, R. (2016). Crítica del Turismo Accesible. Desvelando la configuración discursiva y factual de los procesos de interacción entre Turismo y Discapacidad [Tesis de doctorado, Universidad Miguel Hernández].

(7) Domínguez, T., Darcy, S., \& Alén, E. (2015). Competing for the disability tourism market a comparative exploration of the factors of accessible tourism competitiveness in Spain and Australia. Tourism Management, 47(1), 261-272.

(8) Edgell, D. L. (2019). Managing Sustainable Tourism: A Legacy for the Future. Routledge.

(9) Fernández, M. T. (2007). Turismo accesible: análisis de la accesibilidad hotelera en la provincia de Cádiz [Tesis de doctorado, Universidad de Cádiz].

(10) Gillovic, B., \& McIntosch, A. (2020). Accessibility and Inclusive Tourism Development: Current State and Future Agenda. Sustainability, 12(22), 9722. https://doi.org/10.3390/su12229722

(11) Gillovic, B., McIntosh, A., Darcy, S., \& Cockburn-Wootten, C. (2018). Enabling the language of accessible tourism. Journal of Sustainable Tourism, 26(4), 615-630. https://doi.org/10.1080/09669582.2017.1377209

(12) Gordillo, J. G. y Pérez, A. V. (2015). Diagnóstico del Turismo Accesible para personas con capacidades motrices restringidas en la ciudad de Ibarra, provincia de Imbabura [Tesis de pregrado, Universidad Técnica del Norte].

(13) Guamán-Guevara, A. R., Guamán-Guevara, M. D. y Mancheno-Saá, J. P. (2019). Análisis del turismo accesible en la industria hotelera en la ciudad de Ambato. Dominio de las Ciencias, 5(2), 28-43. http://dx.doi.org/10.23857/dom.cien.pocaip.2019.5.2.abril.28-43

(14) Guerrero, P. (2018). ¿Cómo medir la accesibilidad turística? Importancia de los sistemas de indicadores para validar destinos turísticos accesibles. Tierra Infinita, 4(1), 106-118. https:// doi.org/10.32645/26028131.785

(15) Hernández, R., Fernández, C. y Baptista, M. (2014). Metodología de la investigación (6ª ed.). Mc Graw Hill Education.

(16) Izaguirre, S. y Torres, K. (2017). Accesibilidad hotelera en el municipio de ciudad Valles, S.L.P. II Congreso Virtual Internacional Desarrollo Económico, Social y Empresarial en Iberoamérica.

(17) Marcos, D. y González, D. J. (2003). Turismo accesible "Hacia un turismo para todos". MAZARS. 
(18) Martínez, M. J., y Boujrouf, S. (2018). Turismo accesible, turismo para todos. Análisis de la accesibilidad en los establecimientos hoteleros de Marrakech. Revista Contribuciones a las Ciencias Sociales. $\quad$ http://www.eumed.net/rev/cccss/2018/02/turismo-accesiblemarrakech.html

(19) Martos, M. (2012). Destinos turísticos accesible: Herramientas para mejorar la accesibilidad. Anales de Geografía, 32 (2), 297-321. https://doi.org/10.5209/rev_AGUC.2012.v32.n2.39722 (20) Medina, M. E. (2017). Propuesta de desarrollo del turismo accesible en la reserva de biósfera Isla de Ometepe (Nicaragua). PASOS. Revista de Turismo y Patrimonio Cultural, 15(4), 91324.

(21) Organización Mundial del Turismo [OMT]. (2014). Recomendaciones de la OMT para un turismo Responsable para todos. Organización Mundial del Turismo.

(22) Organización Mundial del Turismo [OMT]. (2015). Manual sobre Turismo Accesible para todos: Principios, herramientas y buenas prácticas - Módulo IV: Indicadores para el estudio de la accesibilidad en el turismo. Organización Mundial del Turismo.

(23) Porto, N. y Rucci, A. C. (2019). Accesibilidad en turismo: Diagnóstico, voluntad política y acciones. Estudios y Perspectivas en Turismo, 28(4), 1032-1062.

(24)Rodríguez, F. M. (2017). Accesibilidad en los establecimientos hoteleros de Almuñécar. La ciudad Accesible. Revista Científica sobre Accesibilidad Universal, 9, 109-143.

(25) Salinas, E., Salinas, E. y Mundet, L. (2019). El Turismo en Cuba: desarrollo, retos y perspectivas. Rosa Dos Ventos Turismo e Hospitalidade, 11(1). http://www.ucs.br/etc/revistas/index.php/rosadosventos/article/view/6249

(26) Sánchez, J., Loarte, M. y Caisachana, D. (2020). Turismo accesible e inclusivo en el Ecuador, frente al turismo accesible en otros países. Universidad y Sociedad, 12(1), 225-231.

(27) Sanz, M. Y. (2018). La inclusión de las personas con discapacidad a las actividades físicorecreativas turísticas en villa clara. Ciencia y Actividad Física, 5(2), 36-47. http://revistaciaf.uclv.edu.cu/index.php/CIAF/article/view/86

(28) Suriá, R. y Escalona, J. Y. (2014). Integración, turismo y discapacidad: ¿son accesibles los hoteles para las personas con discapacidad física? PASOS. Revista de Turismo y Patrimonio Cultural, 12(1), 209-218. https://doi.org/10.25145/j.pasos.2014.12.016

(29) Tite, G., Carrillo, D. y Ochoa, M. (2021). Turismo accesible: estudio bibliométrico. Turismo y Sociedad, 28, 115-132. https://doi.org/10.18601/01207555.n28.06 
(30) Tite, M. (2019). Caracterización de personas con discapacidad e incidencia del turismo accesible en la provincia de Tungurahua, Ecuador. Revista Digital Latioamericana de Turismo Inclusivo Divulgación Académica, (3). https://issuu.com/revista. turismo.inclusivo/docs/rlti_03_divulgaci_n_acad_mica

(31) Travel Trade Caribbean. (2019). Cuba se prepara para ser un destino accesible. https://www.traveltradecaribbean.es/cuba-se-prepara-para-ser-un-destino-accesible/

(32)Zurita, C. (2018). Diagnóstico e inclusión educativa de los niños, adolescentes y jóvenes con necesidades educativas especiales. Editorial Pueblo y Educación.

Cómo citar este artículo: Hernández, F., Hernández, Y. y Rodríguez, M. (2021). Hacia un turismo inclusivo en los hoteles comercializados por Ecotur en La Habana y Varadero. Tendencias, 22(2), 76-106. https://doi.org/10.22267/rtend.212202.169 
Anexo

Ficha técnica utilizada para el diagnóstico de la accesibilidad en las instalaciones hoteleras objeto de estudio

\begin{tabular}{|c|l|l|}
\hline \multicolumn{2}{|l|}{ 1- Accesibilidad Exterior } \\
\hline 1.1 & ¿El establecimiento se encuentra claramente señalizado? & \\
\hline 1.2 & $\begin{array}{l}\text { ¿El recorrido exterior hasta el acceso es adecuado (ancho mínimo de } 1,5 \mathrm{~m} . \mathrm{y} \\
\text { recorrido sin desniveles)? }\end{array}$ \\
\hline 1.3 & ¿Existen plazas de aparcamiento reservadas para personas con movilidad reducida? & \\
\hline 1.4 & $\begin{array}{l}\text { ¿Existen como mínimo un porcentaje equivalente al } 2 \% \text { del total de plazas habilitadas } \\
\text { con dimensiones mínimas de 3,30 x 5,0 m? }\end{array}$ \\
\hline 1.5 & ¿Las dimensiones mínimas de las plazas son de 3,3 x $5 \mathrm{~m} ?$ & \\
\hline 1.6 & ¿Las plazas de aparcamiento para personas con discapacidad están señalizadas? & \\
\hline
\end{tabular}

\section{2- Acceso Principal al Recinto}

\begin{tabular}{|c|l|l|}
\hline 2.1 & ¿Existe algún escalón o desnivel para acceder al establecimiento? & \\
\hline 2.2 & ¿En caso de existir escalones sobrepasan los 12? \\
\hline 2.3 & $\begin{array}{l}\text { ¿En caso de tener escalera la misma es cómoda y segura? (anchura, barandillas, rellanos } \\
\text { adecuados...) O no la necesita }\end{array}$ & \\
\hline 2.4 & $\begin{array}{l}\text { ¿Dispone de rampa? En caso afirmativo ¿Es cómoda y segura? (anchura, pendiente, } \\
\text { embarque, barandillas, rellanos adecuados...) ¿O no la necesita? }\end{array}$ & \\
\hline 2.5 & ¿Existe plataforma elevadora? ¿O no la necesita? \\
\hline 2.6 & $\begin{array}{l}\text { ¿La puerta de acceso al establecimiento tiene una anchura igual o superior a 80 cm o es } \\
\text { abierta? }\end{array}$ & \\
\hline 2.7 & $\begin{array}{l}\text { ¿La puerta es deslizante automática con apertura y cierre doblemente controlado con un } \\
\text { dispositivo de mantener abierta o es abierta? }\end{array}$ \\
\hline 2.8 & $\begin{array}{l}\text { ¿Dispone de mostrador de recepción con dos alturas diferentes o altura adecuada } \\
\text { (máximo 80 cm)? }\end{array}$ \\
\hline $3-$ Comunicación Vertical \\
\hline 3.1 & $\begin{array}{l}\text { ¿El establecimiento tiene planta única o las habitaciones adaptadas y servicios comunes } \\
\text { se encuentran en la planta de acceso? }\end{array}$ \\
\hline 3.2 & $\begin{array}{l}\text { ¿Dispone de escaleras interiores cómodas y seguras (¿anchura, barandilla, descansos?) } \\
\text { o no existen? }\end{array}$ \\
\hline 3.3 & $\begin{array}{l}\text { ¿Dispone de ascensor con tamaño mínimo de 1,10 x 1,4 m y puerta de entrada con ancho } \\
\text { libre mínimo de 0,8 m o no es necesario? }\end{array}$ \\
\hline 3.4 & $\begin{array}{l}\text { ¿El ascensor tiene acceso a todas las plantas donde están las habitaciones adaptadas o } \\
\text { no es necesario? }\end{array}$ \\
\hline 3.5 & ¿Existen espacios o áreas comunes sin acceso en ascensor? \\
\hline 3.6 & \begin{tabular}{l} 
¿El ascensor tiene capacidad para una camilla? \\
\hline 3.7
\end{tabular} & $\begin{array}{l}\text { ¿El ascensor dispone de facilidades para ciegos? (botonera en braille, información } \\
\text { acústica...) }\end{array}$ \\
\hline 3.8 & $\begin{array}{l}\text { ¿El ascensor dispone de facilidades para sordos? (Indicador visual de planta, } \\
\text { intercomunicador accesible...) }\end{array}$ \\
\hline 4- Comunicación Sensorial \\
\hline
\end{tabular}


Hacia un turismo inclusivo en los hoteles comercializados por Ecotur en La Habana y Varadero Félix Hernández Domínguez; Yoan Hernández Flores; Maité Rodríguez González

\begin{tabular}{|c|c|}
\hline 4.1 & ¿La iluminación natural y artificial general del establecimiento es adecuada? \\
\hline 4.2 & $\begin{array}{l}\text { ¿Existe señalización escrita y gráfica que indique las diferentes estancias del } \\
\text { establecimiento? }\end{array}$ \\
\hline 4.3 & $\begin{array}{l}\text { ¿Existe información del establecimiento y sus servicios para personas ciegas? } \\
\text { (cartas en braille, plafones táctiles...) }\end{array}$ \\
\hline 4.4 & $\begin{array}{l}\text { ¿Existen ayudas técnicas para personas sordas? (teléfono de texto, fax, bucles } \\
\text { magnéticos, despertador vibrador, etc....) }\end{array}$ \\
\hline 4.5 & ¿Tienen personal con conocimiento de lengua de signos? \\
\hline \multicolumn{2}{|c|}{ 5- Áreas Comunes } \\
\hline \multicolumn{2}{|c|}{ Zonas de paso o recorridos } \\
\hline 5.1 & ¿Existen escalones aislados o desniveles en las plantas o recorridos del hotel? \\
\hline 5.2 & ¿Existen rampas o plataformas elevadoras para salvar los desniveles o escalones? \\
\hline 5.3 & ¿Existen espacios o áreas comunes sin acceso debido a escalones o desnivel? \\
\hline 5.4 & ¿Los pasillos permiten el cruce de dos personas simultáneamente? \\
\hline 5.5 & ¿El pavimento es antiresbalante? \\
\hline \multicolumn{2}{|c|}{ Puertas } \\
\hline 5.6 & ¿Tienen una anchura mínima de $0 ? 8 \mathrm{~m} ?$ \\
\hline 5.7 & ¿Disponen de placas informativas? \\
\hline 5.8 & ¿Están señalizadas en braille o alto relieve? \\
\hline \multicolumn{2}{|c|}{ Baño Común } \\
\hline 5.9 & ¿Dispone de aseo adaptado en las zonas comunes? \\
\hline 5.10 & ¿Está situado en planta baja con recorrido accesible o en planta accesible? \\
\hline 5.11 & ¿Existe señalización específica de baño adaptado en la puerta? \\
\hline 5.12 & $\begin{array}{l}\text { ¿La puerta y su dimensión es adecuada para su uso por una persona usuaria de silla de } \\
\text { ruedas? }\end{array}$ \\
\hline 5.13 & ¿El baño dispone de ayudas técnicas (barras)? \\
\hline \multicolumn{2}{|c|}{ Cafetería / Restaurante } \\
\hline 5.14 & ¿Dispone de Cafetería / Restaurante? \\
\hline 5.15 & ¿Dispone de barra con dos alturas diferentes? \\
\hline 5.16 & ¿Dispone de alguna mesa con altura entre 75 y $80 \mathrm{~cm} ?$ \\
\hline 5.17 & ¿Los ámbitos de paso principales permiten el cruce de dos personas simultáneamente? \\
\hline 5.18 & $\begin{array}{l}\text { ¿Dispone de menús específicos para Celíacos? (alérgicos al gluten) o Existe posibilidad } \\
\text { de menús diferenciados? }\end{array}$ \\
\hline \multicolumn{2}{|r|}{ Piscina } \\
\hline 5.19 & $\begin{array}{l}\text { Si el establecimiento tiene piscina, dispone de rampa, grúa o plataforma para facilitar el } \\
\text { acceso a personas con discapacidad física o movilidad reducida al paso de la piscina o } \\
\text { la piscina es nivel } 0\end{array}$ \\
\hline \multicolumn{2}{|r|}{ Protección contra incendios y emergencia } \\
\hline 5.20 & $\begin{array}{l}\text { ¿Existen un plan de emergencia que contemple la evacuación de personas con } \\
\text { discapacidad? }\end{array}$ \\
\hline 5.21 & ¿Existe alarma sonora y luminosa? \\
\hline \multicolumn{2}{|c|}{ 6. Habitaciones } \\
\hline 6.1 & ¿Dispone de habitaciones adaptadas para personas con discapacidad? \\
\hline 6.2 & ¿Dispone de 1 habitación adaptada por cada 20 habitaciones corrientes? \\
\hline
\end{tabular}




\begin{tabular}{|c|l|c|}
\hline 6.3 & $\begin{array}{l}\text { ¿La dimensión de la habitación adaptada y su puerta en comparación con la habitación } \\
\text { estándar es mayor? }\end{array}$ & \\
\hline 6.4 & ¿La habitación adaptada dispone de baño adaptado? & \\
\hline 6.5 & ¿La dimensión del baño y su puerta permite su uso a personas usuarias de silla de ruedas? & \\
\hline 6.6 & ¿El baño dispone de ayudas técnicas (barras)? & \\
\hline 6.7 & ¿El baño dispone de ducha? & \\
\hline 6.8 & ¿La ducha cumple con los requisitos para personas en silla de ruedas? & \\
\hline 6.9 & $\begin{array}{l}\text { ¿Dispone la habitación de instalaciones domóticas? (instalaciones inteligentes, } \\
\text { automáticas) }\end{array}$ & \\
\hline 6.10 & ¿Las puertas están bien señalizadas? (números grandes, bien contrastados, etc.) & \\
\hline 6.11 & ¿Las puertas tienen señalización en braille o alto relieve? & \\
\hline 7- Formación & $\begin{array}{l}\text { ¿Disponen de personal que haya recibido formación en la atención a personas con } \\
\text { discapacidad? }\end{array}$ & \\
\hline 8- Clientes & ¿Reciben habitualmente clientes con discapacidad? \\
\hline 8.1 & \\
\hline
\end{tabular}

\section{Observaciones:}

- Ítems relativos a la movilidad reducida: 1.1, 1.2, 1.3, 1.4, 1.5, 2.1, 2.2, 2.3, 2.4, 2.5, 2.6, 2.7, 2.8, 3.1, 3.2, 3.3, 3.4, 3.5, 3.6, 5.1, 5.2, 5.3, 5.4, 5.5, 5.6, 5.7, 5.9, 5.10, 5.11, 5.12, 5.14, 5.15. $5.16,5.19,5.20,6.1,6.2,6.3,6.4,6.5,6.6,6.7,6.8,6.9,7.1$

- Ítems relativos a la discapacidad auditiva: $1.1,3.8,4.4,4.5,5.7,5.14,5.20,5.21,6.1,6.2$, $6.9,7.1$

- Ítems relativos a la discapacidad visual: $1.1,1.2,2.1,2.2,2.3,2.4,2.7,3.1,3.2,3.4,3.5,3.7$, $4.1,4.3,5.1,5.2,5.7,5.8,5.14,5.20,5.21,6.1,6.2,6.9,6.10,6.11,7.1$

- Ítems relativos a la discapacidad cognitiva: 1.1, 3.2, 4.2, 5.7, 5.14, 5.20, 6.1, 6.2, 6.9, 6.10, 7.1 